\title{
Single-center evaluation of a next generation fully repositionable and retrievable transcatheter aortic valve replacement
}

\author{
Karolina Berntorp* ${ }^{*}$, Sasha Koul, Shahab Nozohoor, Jan Harnek, Henrik Bjursten and Matthias Götberg
}

\begin{abstract}
Background: The mechanically expandable Lotus Valve System is a fully repositionable and retrievable valve with an adaptive seal to minimize paravalvular leak (PVL). The aim of this study was to evaluate the short- and long-term safety and efficacy of the new device with focus on a new implantation technique to reduce the need for a permanent pacemaker (PPM) post procedure.

Methods: We performed a prospective single-center, non-randomized evaluation of the Lotus Valve System. The first 100 consecutive Lotus Valve implantations were included in the analysis. Outcome was assessed according to VARC2-criteria. Postoperative pacemaker rates were assessed using the national pacemaker registry and electronic medical records. Mortality at 30 days and 12 months were acquired from the national population registry.

Results: Mean age was $82.7 \pm 5.6$ years, mean Euroscore I was $25.3 \pm 14.5 \%$, mean STS-score was $6.5 \pm 4.1 \%$ and mean aortic valve area was $0.6 \pm 0.1 \mathrm{~cm}^{2}$. There were no cases of valve embolization, ectopic valve deployment or additional valve implantation. Device success according to the VARC2-criteria was $97 \%$. The 30-day mortality rate was 3\%. Two deaths occurred due to stroke and one due to a ventricular rupture. Major stroke rate was 2\% and major vascular complication rate was $2 \%$. The 12 -month mortality rate was $14 \%$. At discharge $87 \%$ of patients had no/trace PVL, $12 \%$ had mild PVL and one patient had a moderate PVL. A total of 13\% received a new PPM post valve implantation. Among patients who did not have a PPM before the procedure, the PPM rate was 15.3\%.
\end{abstract}

Conclusions: This single-center evaluation of the Lotus Valve System demonstrated a good clinical outcome with a low mortality, in a high-risk population. Introduction of a new implantation technique resulted in lower PPM rates than previously reported without negatively affecting PVL.

Trial registration: Current Controlled Trials ISRCTN14952278, retrospectively registered 06/11/2017.

Keywords: Transcatheter aortic valve replacement, Paravalvular leak, Permanent pacemaker

\section{Background}

Transcatheter Aortic Valve Implantation (TAVI) has become a recommended alternative for severe symptomatic aortic stenosis in patients deemed to be in intermediate or high risk for surgical aortic valve replacement (SAVR), and is standard therapy when surgery is denied. Compared with SAVR, TAVI provides similar or improved outcome in large randomized clinical studies involving primarily

\footnotetext{
* Correspondence: karolina.berntorp@med.lu.se

Department of Cardiology, Clinical Sciences, Lund University, Skane University Hospital, 221-85 Lund, SE, Sweden
}

patients deemed at intermediate or high risk for SAVR [1-6].

The new mechanically expandable Lotus Valve System enables a precise positioning due to the ability to reposition or fully retrieve the valve even in a locked final state before detaching the bioprosthetic valve from the delivery catheter. The Lotus Valve System also has an adaptive seal to potentially reduce paravalvular leak (PVL) $[7,8]$. Reducing PVL is an important feature of modern valves since residual moderate or severe PVL after valve implantation

(c) The Author(s). 2019 Open Access This article is distributed under the terms of the Creative Commons Attribution 4.0 International License (http://creativecommons.org/licenses/by/4.0/), which permits unrestricted use, distribution, and reproduction in any medium, provided you give appropriate credit to the original author(s) and the source, provide a link to the Creative Commons license, and indicate if changes were made. The Creative Commons Public Domain Dedication waiver (http://creativecommons.org/publicdomain/zero/1.0/) applies to the data made available in this article, unless otherwise stated. 
has been demonstrated to be an important negative predictor of long-term outcome $[9,10]$.

A well-known adverse event to TAVI is the need of a permanent pacemaker (PPM) after implantation due to mechanical interaction of the valve on the electrical conduction system, involving the atrioventricular (AV) node and/or left bundle, causing 2nd degree or 3rd degree AV block [1, 8, 11]. Available evidence on predictors of a PPM implantation are derived from small studies but 1st degree AV block, left anterior hemiblock (LAH) and right bundle branch block (RBBB) have been suggested $[12,13]$. The Lotus Valve System has been associated with a relatively high incidence of PPM ranging from 24 to $36 \%$ in different studies $[7,8,11,14]$. After gaining initial experience, we introduced a new implantation technique to address the high PPM rate. The technique was based on avoiding valve oversizing relative to the annulus, potentially minimizing the interaction between the valve and the left ventricular outflow tract (LVOT). Furthermore, we actively sought a high implantation of the valve in a more aortic position, minimizing interaction with the electrical conduction system.

We evaluated the procedural, short- and long-term safety and efficacy of the Lotus Valve System in an all-comers population with severe symptomatic aortic stenosis at our center, according to the Valve Academic Research Consortium - 2 (VARC-2) criteria [15].

\section{Methods}

\section{Study design}

We prospectively collected information with the intent to evaluate the safety and efficacy of the mechanically expandable Lotus Valve System (Boston Scientific, Marlborough, MA, USA) in the first 100 patients with a Lotus Valve implanted at Skåne University Hospital, Lund, Sweden. The Lotus Valve System was used in an all-comers patient cohort, after heart team acceptance when deemed anatomically feasible. Limiting factors for use of the Lotus Valve was an annulus $>27 \mathrm{~mm}$ and inadequate vascular access precluding transfemoral approach. The primary outcome measure was device success according to the VARC-2 criterion defined as the absence of procedural mortality and correct positioning of a valve. The secondary outcomes were all-cause mortality, major stroke, major vascular bleeding and a new PPM before discharge, according to the European Society of Cardiology (ESC) guidelines [16]. The study was retrospectively registered at current Controlled Trials ISRCTN14952278, $06 / 11 / 2017$.

\section{Patient selection, procedure and follow-up}

All patients were assessed to have severe symptomatic aortic stenosis with an indication for TAVI by two multidisciplinary heart team meetings consisting of at least one cardiologist and a cardiac surgeon. Patient risk for surgical valve replacement was based on the Society of Thoracic Surgeons (STS) score for mortality and Euroscore I. All patients went through a pre-procedural angiography and electrocardiogram (ECG)-gated computed tomography for valve sizing and assessment of vascular access. Three different independent experienced cardiologists performed transthoracic echocardiogram (TTE) measurements. PVL and measurements of mean aortic gradient and left ventricular ejection fraction (LVEF) were performed on all patients before discharge. PVL was graded into no/trace, mild, moderate and severe as described elsewhere [15]. Follow-up was conducted according to local clinical practice at the referring hospital. TTE was not performed by a central core lab.

The proctored implantation technique of the Lotus Valve System involves early flaring of the lower portion of the valve in a deep position in the LVOT followed by pulling on the catheter, seating the Lotus Valve into the final position as it expands. After gaining initial experience, we utilized a modified technique which involved limited flaring of the Lotus Valve before seating the valve in a more aortic position with the aim of a final implantation depth of $0-6 \mathrm{~mm}$, or in other words never to allow the frame to come deeper than $0-6 \mathrm{~mm}$ into the LVOT. However, this was done without allowing the cranial part of the Lotus Valve frame to cover the coronary ostia. With this technique the bottom of the Lotus Valve seldom reached $10 \mathrm{~mm}$ below the annulus during implant.

\section{Data sources and statistical analysis}

This study conforms to the CONSORT guidelines. The pre- and post-operative data were retrieved from electronic medical records. All-cause mortality rate was assessed using the national population registry, which has a 100\% follow-up of all citizens. PPM rates were assessed using the national pacemaker registry and electronic medical records. Continuous variables are presented as mean \pm standard deviation (SD). Categorical variables are presented as counts and percentages. Comparisons were made using t-test for continuous variables and the chi-square test for categorical variables. A $p$-value $<0.05$ was considered statistically significant. For statistical analysis the IBM SPSS Statistics 23 was used.

\section{Results \\ Patients}

Between September 2013 to November 2015, 100 patients with an implanted Lotus Valve System were included. Baseline, procedural and follow-up data at 30-days were available for all patients (Tables 1,2,3). The mean age was $82.7 \pm 5.6$ years with $44 \%$ being male. The mean Euroscore I was $25.3 \pm 14.5 \%$, mean STS-score was $6.5 \pm 4.1 \%$. The mean aortic valve area at baseline was $0.6 \pm 0.1 \mathrm{~cm}^{2}$ 
Table 1 Patient characteristics at baseline $(N=100)$

\begin{tabular}{ll}
\hline Age - yrs. mean ( \pm SD) & $82.7(5.6)$ \\
Female sex - n (\%) & $56(56)$ \\
Medically treated diabetes - n (\%) & $30(30)$ \\
Arterial hypertension - n (\%) & $91(91)$ \\
Atrial fibrillation - n (\%) & $42(42)$ \\
Previous Percutaneous Coronary Intervention - n (\%) & $23(23)$ \\
Previous Coronary Artery Bypass Grafting - n (\%) & $22(22)$ \\
Previous stroke - n (\%) & $14(14)$ \\
Chronic renal failure - n (\%) & $33(33)$ \\
Pulmonary hypertension (severe) - n (\%) & $28(28)$ \\
Porcelain aorta - n (\%) & $16(16)$ \\
New York Heart Association functional class III or IV - n (\%) & $79(79)$ \\
Society of Thoracic Surgeons score - \% mean ( \pm SD) & $6.5(4.1)$ \\
Euroscore - \% mean ( \pm SD) & $25.3(14.5)$ \\
Permanent pacemaker at baseline - n (\%) & $15(15)$ \\
\hline
\end{tabular}

(Table 4). The majority of patients (79\%) were in New York Heart Association (NYHA) class III or IV.

\section{Device failure and secondary outcomes}

Transfemoral approach was used in $95 \%$ of the implants and $5 \%$ had a transaortic approach. Conscious sedation was used in $54 \%$ of patients (Table 2). One patient planned for a Lotus Valve System was converted to an Edwards Sapien 3 valve due to severe aortic tortuosity with inability to track the delivery catheter. In one patient a device failure occurred when a reposition was performed. The device was successfully retrieved but with a femoral access complication. The patient received a new Lotus Valve System 42 days later (Table 3). There were two cases of major vascular complication, requiring surgical intervention. There were no cases of coronary obstruction, valve embolization, ectopic valve deployment or additional valve implantation (Table 3). The procedural mortality rate was $1 \%$ due to a ventricular rupture in a patient who had been treated with steroids

Table 2 Procedural data $(N=100)$

\begin{tabular}{ll}
\hline & Percent \\
\hline Transfemoral approach & 95 \\
Transaortic approach & 5 \\
Bicuspid aortic stenosis & 3 \\
Valve in valve procedure & 1 \\
Severe aortic regurgitation & 1 \\
General anesthesia & 46 \\
Predilatation & 48 \\
Postdilatation & 1 \\
Prothesis size distribution 23/25/27 mm & $32 / 29 / 39$ \\
\hline
\end{tabular}

Table 3 Procedural and clinical outcome

\begin{tabular}{ll}
\hline & $\mathrm{n} / \mathrm{N}(\%)$ \\
\hline Device success & $99 / 102(97)^{\mathrm{a}}$ \\
Successful valve retrieval if attempted & $2 / 2(100)$ \\
Coronary obstruction & $0 / 100(0)$ \\
Valve migration & $0 / 100(0)$ \\
Valve embolization & $0 / 100(0)$ \\
Ectopic valve deployment & $0 / 100(0)$ \\
TAVI-in-TAV deployment & $0 / 100(0)$ \\
Procedural mortality & $1 / 100(1)$ \\
30 day mortality & $3 / 100(3)$ \\
Major stroke & $2 / 100(2)$ \\
Major vascular complications & $2 / 100(2)$ \\
aAbsence of procedural mortality, successful access, delivery, deployment and \\
system retrieval
\end{tabular}

for many years. In addition, two patients suffered from periprocedural fatal ischemic strokes, probably from calcium dislodgement, resulting in an overall 30-day mortality rate of $3 \%$. All-cause mortality at one year was $14 \%$, representing one new cardiac death, 7 new non-cardiac deaths and three deaths of unknown cause.

\section{Echocardiographic follow-up}

The TTE examination at discharge was performed in 100\% of the patients and LVEF, mean aortic gradient and PVL were measured. One year follow-up TTE examination measurements and follow-up were not available for all patients due to differences in follow-up routines between referring hospitals. After one year TTE measurements were available in $52 \%$ of the patients. The mean aortic gradient was 47.2 $\pm 0.1 \mathrm{mmHg}$ at baseline, $9.9 \pm 3.4 \mathrm{mmHg}$ at discharge and $12.2 \pm 4.6 \mathrm{mmHg}$ at one year, $p=0.08$ (Table 4). The mean LVEF was $44.1 \pm 11.1 \%$ at baseline, $48.9 \pm 9.6 \%$ at discharge and $50.5 \pm 7.9 \%$ at one year, $p=0.17$.

The rate of PVL after TAVI was no/trace in $87 \%$ and mild in $12 \%$ of the patients at discharge (Table 4 ). One patient had a moderate PVL due to an annular calcified nodule $(9 \times 7 \mathrm{~mm})$. Similar rates of PVL were observed at one year with no/trace in $94.1 \%$ and mild in $3.9 \%$ of patients. One patient presented 5 months post TAVI with a severe PVL due to an aortic root abscess. The patient was successfully treated by antibiotics with good outcome.

\section{Pacemaker and conduction disturbances}

Before implantation $15 \%$ of the patients had a preexisting pacemaker. After implantation, but before discharge, 13 patients (13\%) received a new PPM according to ESC guidelines. Among patients who did not have a PPM prior to TAVI, the PPM rate was $15.3 \%$ (13/85 patients). The majority of patients $(12 / 13)$ received a PPM due to peri- or post-procedural 3rd degree AV block and one 
Table 4 Echocardiogram characteristics at baseline, discharge and 1 year

\begin{tabular}{|c|c|c|c|c|}
\hline & Baseline ( \pm SD) & Discharge $( \pm$ SD) & 1 year $( \pm S D)^{a}$ & $p$-value 1 year compared to discharge \\
\hline Mean LVEF \% & $44.05(11.1)$ & $48.9(9.6)$ & $50.5(7.9)$ & 0.17 \\
\hline Mean aortic gradient mmHg & $47.2(0.1)$ & $9.9(3.4)$ & $12.2(4.6)$ & 0.08 \\
\hline \multirow[t]{2}{*}{ Mean aortic valve area $\mathrm{cm}^{2}$} & $0.6(0.1)$ & - & - & \\
\hline & Baseline & Discharge & 1 year $^{a}$ & $p$-value 1 year compared to discharge \\
\hline PVL & & & & 0.06 \\
\hline None/Trace \% & - & 87 & 90.2 & \\
\hline Mild \% & - & 12 & 5.9 & \\
\hline Moderate $\%$ & - & 1 & 0 & \\
\hline Severe \% & - & 0 & 2 & \\
\hline
\end{tabular}

${ }^{a}$ One year follow-up data were available for $52 \%$ of the patients

due to 2nd degree type II AV block. After one year 4 more patients had received a new PPM. Three patients due to 3rd degree AV block and one due to an unspecified AV block.

There were 37 patients who developed a LBBB (left bundle branch block) post TAVI. Among the patients who had a PPM implanted after discharge their discharge ECG were as follows; two patients had a 1st degree AV block and LBBB, one had a 1st degree AV block and incomplete LBBB and in one patient the discharge ECG was missing.

\section{Discussion}

Our single-center study demonstrated a high rate of device success with good clinical outcome in a high-risk population with severe symptomatic aortic stenosis receiving the mechanically expandable Lotus Valve System. We also reported low rates of new PPM using a modified implantation technique together with low rates of major stroke, major vascular bleeding and PVL. The ability to fully reposition or retrieve the valve, even in a locked final position meant that we had no cases of valve migration, valve embolization or TAVI-in-TAV deployment.

The need of a PPM after TAVI has not been associated with any increased risk of mortality. [17, 18] It does however add costs of another invasive procedure with associated risk of complications, and delays in patient discharge. High PPM rates after implantation of a Lotus Valve System compared to 1st generation TAVI has been identified as a significant drawback of the technology. $[19,20]$ The PPM rates in all patients in the REPRISE I and II studies were 36 and $28.6 \%$ respectively. $[7,8]$ Two single-center studies using the Lotus Valve System have demonstrated an incidence of new PPM of 24.1 and $39.9 \%$ in pacemaker-naïve patients. $[11,20]$ A third single-center study presented $28 \%$ PPM rate in all patients. [21] Furthermore, the RESPOND post market evaluation study demonstrated $30 \%$ new PPM in all patients and $34.6 \%$ in pacemaker-naïve patients after implantation of the Lotus Valve System. [14] Krackhardt et al. have previously reported 9.5\% PPM in a small case series after using a high implantation technique. [22] We reported 13\% PPM rate in all patients due to 2nd degree type II or 3rd degree AV block, and $15.3 \%$ in pacemaker-naïve patients. We have demonstrated low PPM rate confirming the benefit of limiting the implantation depth. In the Swedish Percutaneous Valve Registry, where all TAVI in Sweden are registered, the combined reported PPM rate after Lotus Valve implantation in all implanting hospitals in Sweden has been less than $17 \%$ since 2013. [23] A possible explanation for the observed low PPM-rates in this study is a careful pre-operative assessment using gated computer tomography to avoid oversizing the Lotus Valve, and the introduction of a new implantation technique where the valve is kept in a high position during the entire deployment aiming to avoid mechanical interaction with the LVOT. Ideal final Lotus Valve position was considered to be with minimal valve protrusion in LVOT while still allowing access of the coronary ostia.

The Lotus Valve System was designed to reduce PVL compared to 1st generation TAVI valves by using an adaptive seal. $[19,20]$ The occurrence of moderate or severe PVL after TAVI implantation is an independent predictor of increased short and long-term mortality rate. [9, 10, 24, 25] In the REPRISE II CE mark multicenter study where 120 patients received a Lotus Valve System, the rate of PVL at discharge was mild in $13.1 \%$ and moderate in $2 \%$ of patients. After one year $11.4 \%$ of patients had a mild PVL and no patient had a moderate or severe PVL. [8] In a single-center experience including 110 patients there were no patients with moderate/severe PVL, with mild PVL noted in 9.1\%. [11] In another single-center experience including 50 Lotus patients moderate/severe PVL was $2 \%$ and mild 6\%. [21] The corresponding numbers in a newly published single-center experience with 202 Lotus Valves were 0 and 3\% respectively. [20] Theoretically, using an implantation technique in which the Lotus Valve interaction with the LVOT is minimized, a higher incidence of PVL could potentially be expected due to less engagement of the adaptive seal in the LVOT. However, we show similar 
results to other studies with $12 \%$ mild PVL and only one case of moderate PVL due to a calcified nodule. This suggests that the modified technique utilized in this patient cohort did not negatively affect PVL.

There are several limitations to this study. First, this was a single-center non-randomized study. Second, TTE measurements were not performed by a central core lab, and follow-up TTE were not available in all patients. The main findings of this small study need to be verified in a larger study.

\section{Conclusions}

In our study the Lotus Valve System demonstrated a high rate of device success and a low rate of procedural mortality, with low rates of procedural complications in a high-risk patient population with severe symptomatic aortic stenosis. By avoiding oversizing the Lotus Valve and introducing a new implantation technique we achieved a low need for new PPM without negatively affecting PVL. These results may provide insight on how to optimize implantation when using the Lotus Valve System.

\section{Abbreviations}

AV: Atrioventricular; ECG: Electrocardiogram; ESC: the european society of cardiology; LAH: Left anterior hemiblock; LBBB: Left bundle branch block; LVEF: Left ventricular ejection fraction; LVOT: Left ventricular outflow tract; PPM: Permanent pacemaker; PVL: Paravalvular leak; RBBB: Right bundle branch block; SAVR: Surgical aortic valve replacement; STS: the society of thoracic surgeons; TAVI: Transcatheter aortic valve implantation; TTE: Transthoracic echocardiogram; VARC: the valve academic research consortium

\section{Acknowledgements}

Not applicable.

\section{Funding}

This research was funded by a Skane University Hospital research fund. This fund had no role in design of the study, collection, analysis, and interpretation of data or writing of the manuscript.

\section{Availability of data and materials}

The data was collected from the electronic medical records, identical to the data in the SWEDEHEART, which is a national quality registry. Data were also collected from the pacemaker registry. These registries are not publically available and therefore required ethics approval (see Ethics approval and consent to participate). Links for further registry information are available at unw.ucr.uu.se/swedeheart/ and www.pacemakerregistret.se. The collected data is available from the corresponding author.

\section{Authors' contributions}

$H B, M G$ and $J H$ designed the study. JH, HB, KB, MG, SK and SN collected the study data. KB, MG and SK performed the main analyses. All authors were involved in drafting and/or official revision of the manuscript. All authors have given final approval of the manuscript and have agreed to be accountable for all aspects of the work.

\section{Ethics approval and consent to participate}

No specific consent for participation in the registry was needed since the data collected was identical to already available data in SWEDEHEART, which is a national quality registry and does not require consent. The data from the SWEDEHEART and the pacemaker registry is not publically available and therefore required ethics approval which was approved by Lund University ethics committee (LU2009/87), Lund, Sweden. The study as a whole was also approved by Lund University ethics committee (LU2009/87), Lund, Sweden, including administrative permission for obtaining and collecting data as well as medical records.
Consent for publication

Not applicable.

\section{Competing interests}

$H B, M G, J H$ has received consulting fees from Boston Scientific corporation to serve as proctors for the Lotus Valve.

\section{Publisher's Note}

Springer Nature remains neutral with regard to jurisdictional claims in published maps and institutional affiliations.

Received: 24 October 2017 Accepted: 15 February 2019

Published online: 26 February 2019

\section{References}

1. Popma JJ, et al. Transcatheter aortic valve replacement using a selfexpanding bioprosthesis in patients with severe aortic stenosis at extreme risk for surgery. J Am Coll Cardiol. 2014;63(19):1972-81.

2. Adams DH, Popma JJ, Reardon MJ. Transcatheter aortic-valve replacement with a self-expanding prosthesis. N Engl J Med. 2014;371(10):967-8.

3. Reardon MJ, et al. Surgical or Transcatheter aortic-valve replacement in intermediate-risk patients. N Engl J Med. 2017;376(14):1321-31.

4. Smith $C R$, et al. Transcatheter versus surgical aortic-valve replacement in high-risk patients. N Engl J Med. 2011;364(23):2187-98.

5. Leon MB, et al. Transcatheter aortic-valve implantation for aortic stenosis in patients who cannot undergo surgery. N Engl J Med. 2010;363(17):1597607.

6. Leon $\mathrm{MB}$, et al. Transcatheter or surgical aortic-valve replacement in intermediate-risk patients. N Engl J Med. 2016;374(17):1609-20.

7. Meredith IT, et al. Transfemoral aortic valve replacement with the repositionable Lotus valve system in high surgical risk patients: the REPRISE I study. Eurolntervention. 2014;9(11):1264-70.

8. Meredith IT, et al. 1-year outcomes with the fully repositionable and retrievable Lotus Transcatheter aortic replacement valve in 120 high-risk surgical patients with severe aortic stenosis: Results of the REPRISE II Study. JACC Cardiovasc Interv. 2016;9(4):376-84.

9. Gotzmann M., et al., Transcatheter aortic valve implantation in patients with severe symptomatic aortic valve stenosis-predictors of mortality and poor treatment response. Am Heart J, 2011. 162(2): p. 238-245 e1.

10. Abdel-Wahab $\mathrm{M}$, et al. Aortic regurgitation after transcatheter aortic valve implantation: incidence and early outcome. Results from the German transcatheter aortic valve interventions registry. Heart. 2011;97(11):899-906.

11. Wohrle J, et al. Transfemoral aortic valve implantation with the repositionable Lotus valve for treatment of patients with symptomatic severe aortic stenosis: results from a single-Centre experience. Eurolntervention. 2016;12(6):760-7.

12. Erkapic $D$, et al. Risk for permanent pacemaker after transcatheter aortic valve implantation: a comprehensive analysis of the literature. J Cardiovasc Electrophysiol. 2012;23(4):391-7.

13. Siontis GC, et al. Predictors of permanent pacemaker implantation in patients with severe aortic stenosis undergoing TAVR: a meta-analysis. J Am Coll Cardiol. 2014;64(2):129-40.

14. Falk $V$, et al. Safety and efficacy of a repositionable and fully retrievable aortic valve used in routine clinical practice: the RESPOND study. Eur Heart J. 2017.

15. Kappetein AP, et al. Updated standardized endpoint definitions for transcatheter aortic valve implantation: the valve academic research Consortium- 2 consensus document. J Thorac Cardiovasc Surg. 2013;145(1):6-23.

16. Brignole $M$, et al. ESC Guidelines on cardiac pacing and cardiac resynchronization therapy: the Task Force on cardiac pacing and resynchronization therapy of the European Society of Cardiology (ESC) Developed in collaboration with the European Heart Rhythm Association (EHRA). Eur Heart J. 2013;34(29):2281-329.

17. Buellesfeld $L$, et al. Impact of permanent pacemaker implantation on clinical outcome among patients undergoing transcatheter aortic valve implantation. J Am Coll Cardiol. 2012;60(6):493-501.

18. Urena $\mathrm{M}$, et al. Permanent pacemaker implantation after transcatheter aortic valve implantation: impact on late clinical outcomes and left ventricular function. Circulation. 2014;129(11):1233-43.

19. Bjursten $H$, et al. The safety of introducing a new generation TAVR device: one departments experience from introducing a second generation repositionable TAVR. BMC Cardiovasc Disord. 2017;17(1):25. 
20. Seeger J, et al. Outcome with the repositionable and retrievable Boston Scientific Lotus valve compared with the balloon-expandable Edwards Sapien 3 valve in patients undergoing Transfemoral aortic valve replacement. Circ Cardiovasc Interv. 2017;10(6).

21. Gooley RP, et al. Comparison of self-expanding and mechanically expanded Transcatheter aortic valve prostheses. JACC Cardiovasc Interv. 2015;8(7):962-71.

22. Krackhardt $F$, et al. Low permanent pacemaker rates following Lotus device implantation for transcatheter aortic valve replacement due to modified implantation protocol. Cardiol J. 2017;24(3):250-8.

23. Tomas Jernberg, D.H., 18288 Stockholm, SWEDEHEART Annual Report 2017: Percutaneous Valve Registry. 2017: http://www.ucr.uu.se/swedeheart/. p. 204-217.

24. Athappan G, et al. Incidence, predictors, and outcomes of aortic regurgitation after transcatheter aortic valve replacement: meta-analysis and systematic review of literature. J Am Coll Cardiol. 2013;61(15):1585-95.

25. Kodali S, et al. Paravalvular regurgitation after transcatheter aortic valve replacement with the Edwards sapien valve in the PARTNER trial: characterizing patients and impact on outcomes. Eur Heart J. 2015;36(7):449-56.

Ready to submit your research? Choose BMC and benefit from:

- fast, convenient online submission

- thorough peer review by experienced researchers in your field

- rapid publication on acceptance

- support for research data, including large and complex data types

- gold Open Access which fosters wider collaboration and increased citations

- maximum visibility for your research: over $100 \mathrm{M}$ website views per year

At BMC, research is always in progress.

Learn more biomedcentral.com/submissions 